\title{
High-level Plasmodium falciparum sulfadoxine-pyrimethamine resistance with the concomitant occurrence of septuple haplotype in Tanzania
}

Vito Baraka ${ }^{1,2^{*}} \mathbb{D}$, Deus S. Ishengoma ${ }^{1}$, Filbert Fransis' ${ }^{1}$, Daniel T. R. Minja' ${ }^{1}$, Rashid A. Madebe ${ }^{1}$, Deogratius Ngatunga ${ }^{3}$ and Jean-Pierre Van Geertruyden ${ }^{2}$

\begin{abstract}
Background: Tanzania abandoned sulfadoxine-pyrimethamine (SP) as the first-line treatment for uncomplicated malaria in 2006 due to high levels Plasmodium falciparum resistance. However, SP is still being used for intermittent preventive treatment during pregnancy (IPTP-SP). This study aimed to assess the pattern of P. falciparum dihydrofolate reductase (Pfdhfr) and dihydropteroate synthetase (Pfdhps) mutations and associated haplotypes in areas with different malaria transmission intensities in mainland Tanzania, 6 years after withdrawal of SP as a first-line treatment regimen for uncomplicated malaria.

Methods: A total of 264 samples were collected during cross-sectional surveys in three districts of Muheza, Muleba and Nachingwea in Tanga, Kagera and Lindi regions, respectively. Parasite genomic DNA was extracted from $P$. falciparum positive samples. The Pfdhfr, Pfdhps single nucleotide polymorphisms (SNPs) were amplified using nested polymerase chain reaction and detected by sequence specific oligonucleotide probe-enzyme linked immunosorbent assay (SSOP-ELISA).

Results: The prevalence of the mutant Pfdhfr-Pfdhps haplotypes was heterogenous and transmission dependent. The triple Pfdhfr mutant haplotypes (CIRNI) were predominant in all sites with significantly higher frequencies at Muheza (93.3\%) compared to Muleba $(75.0 \%)$ and Nachingwea districts $(70.6 \%),(p<0.001)$. Overall, the prevalence of the wild-type Pfdhps (SAKAA) haplotype was lowest at Muheza $(1.3 \%)$, $(p=0.002)$. Double Pfdhps haplotype SGEAA was significantly high at Muheza (27.2\%) and Muleba (20.8\%) while none (0 \%) was detected at Nachingwea $(p<0.001)$. The prevalence of triple Pfdhps SGEGA haplotype was significantly higher at Muheza compared to Muleba and Nachingwea ( $p<0.001)$. In contrast, Nachingwea and Muleba had significantly higher prevalence of another triple Pfdhps AGEAA haplotype $\left(x^{2}=39.9, p<0.001\right)$. Conversely, Pfdhfr-Pfdhps as quintuple and sextuple haplotypes were predominant including the emergence of a septuple mutant haplotype CIRN|-AGEGA $(n=11)$ observed at Muheza and Muleba.

Conclusion: These results ascertain the high prevalence and saturation of Pfdhfr and Pfdhps haplotypes conferring SP resistance in areas with changing malaria epidemiology; and this could undermine the use of IPTp-SP in improving pregnancy outcomes. In these settings where high level SP resistance is documented, additional control efforts are needed and evaluation of an alternative drug for IPTp is an urgent priority.
\end{abstract}

\footnotetext{
*Correspondence: vitobaraka@gmail.com

${ }^{1}$ National Institute for Medical Research, Tanga Research Centre, P. O.

Box 5004, Tanga, United Republic of Tanzania

Full list of author information is available at the end of the article
} 
Keywords: Plasmodium falciparum, Single nucleotide polymorphisms, Sulphadoxine-pyrimethamine, Molecular markers, Septuple Pfdhfr, Pfdhps, Tanzania

\section{Background}

Plasmodium falciparum malaria remains a major public health problem in the sub-Saharan Africa (SSA). Increased global efforts in malaria control and elimination have resulted into significant reduction in the disease burden through scaling up of control interventions such as use of insecticide-treated nets (ITNs), indoor residual spraying (IRS) and early case diagnosis and prompt treatment using effective anti-malarial drugs [1]. However, malaria control programmes are repeatedly challenged by rapid and widespread anti-malarial drug resistance [2-4]. Following the widespread drug resistance to sulfadoxine-pyrimethamine (SP) and chloroquine (CQ) $[5,6]$, the World Health Organization (WHO) recommended a policy change from monotherapy to artemisinin-based combination therapy (ACT) [7]. Despite policy changes as a result of widespread drug resistance against SP, the drug is still being recommended for intermittent preventive treatment during pregnancy (IPTp$\mathrm{SP}$ ) whereby in areas of moderate to high transmission SP dose is given at each scheduled antenatal care (ANC) visit at least monthly to prevent pregnancy associated malaria (PAM) and improve pregnancy outcomes [8]. In addition, SP is still being used in the IPT in infants (IPTi-SP) to reduce malaria and anaemia among infants as well as seasonal malaria chemoprevention (SMC) programmes in some malaria endemic settings [9]. Nonetheless, the chemoprophylactic effectiveness of IPTp-SP, IPTi-SP and SMC-SP strategies against malaria control amongst the most vulnerable is increasingly being compromised due to the rapid and widespread SP-resistance.

In Tanzania, SP was introduced as the first-line antimalarial drug for treatment of uncomplicated falciparum malaria in 2001 as a result of high level chloroquine (CQ) resistance (CQR) and clinical treatment failures (TFs) [10]. However, 5 years after its introduction, the policy was again revised in November 2006 due to widespread resistance against SP and remarkable TFs $[4,11]$. Thus, artemether-lumefantrine (AL), an ACT, was introduced as the first-line treatment for uncomplicated falciparum malaria in the mainland Tanzania [12]. The changes in malaria treatment policies were supported by data from molecular epidemiological resistance surveillance against CQ and SP. The in vivo molecular surveillance ascertained the treatment failures (TFs) $[4,11]$ with $P$. falciparum resistance against $\mathrm{CQ}$ and $\mathrm{SP}$ in clinical settings.

Sulfadoxine-pyrimethamine acts by inhibiting the P. falciparum dihydrofolate reductase (DHFR) and dihydropteroate synthetase (DHPS) enzymes [13, 14]. Notably, several single nucleotide polymorphisms (SNPs) in the Pfdhps gene at codons Serine 436 to Alanine (S436A), Alanine 437 to Glycine (A437G), Lysine 540 Glutamic acid (K540E), Alanine 581 to Glycine (A581G) and Alanine 613 to Serine (A613S) are associated with sulfadoxine resistance. Pyrimethamine resistance is conferred by mutations in the Pfdhfr gene resulting from amino acid substitution at codons Cysteine 50 to Arginine (C50R), Asparagine 51 to Isoleucine (N51I), Cysteine 59 to Arginine (C59R), Serine 108 to Asparagine/Threonine $(\mathrm{S} 108 \mathrm{~N} / \mathrm{T})$, and Isoleucine 164 to Leucine (I164L) $[15,16]$. Emergence and subsequent accumulation of the mutations in both the Pfdhfr/Pfdhps genes is associated with SP clinical TF in several epidemiological settings [11, 17-19]. The major Pfdhfr haplotypes emerge as a result of combination of mutations of the wildtype cysteine-asparagine-cysteine-asparagine-isoleucine (CNCNI) followed by the gradual changes resulting to cysteine-isoleucinecysteine-asparagine-isoleucine (CICNI), cysteineasparagine-arginine-asparagine-isoleucine (CNRNI), cysteine-isoleucine-arginine-asparagine-isoleucine (CIRNI) and cysteine-isoleucine-arginine-asparagineleucine (CIRNL) as a single, double, triple and quadruple mutants, respectively (at amino acid positions C50R, N51I, C59ㅈ, S108N, and L164II) [20, 21]. For Pfdhps, the wild type genotype serine-alanine-lysine-alanine-alanine (SAKAA) can change to a single alanine-alanine-lysinealanine-alanine or serine-glycine-lysine- alanine-ala-

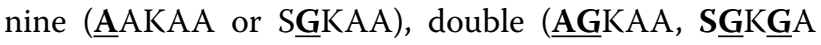
or SGEAA) or triple mutants (AGEAA or SGEGA, at amino acid positions S436 $\underline{\text { A }, ~ A 437 G, ~ K 540 E, ~ A 581 G ~ a n d ~}$ A613 $\underline{\text { S/T) }}[$ [14, 22, 23]. Quintuple mutations as a result of the combination of Pfdhfr triple (CIRNI) and the Pfdhps double (A437G, K540E) mutations were reported in East Africa $[24,25]$ and have been shown to confer high level SP resistance rendering SP ineffective for treatment of $P$. falciparum infections. In northeastern Tanzania, the rise in Pfdhps mutations at codons A581 $\underline{\mathbf{G}}$ has led to higher proportions of SGEGA haplotypes [26]. Combined Pfdhfr-Pfdhps SGEGA-CIRNI forming the sextuple haplotype has been associated with sub-optimal prophylactic effect and thus poor pregnancy outcomes particularly in areas where SP resistance is widespread in sub-Saharan Africa [17, 18, 27].

In fact, WHO recommends stopping IPTp-SP in areas where the Pfdhfr K540E prevalence is $>95 \%$ and Pfdhps A581 is is $>10 \%$ as SP is likely to be ineffective [28]. The 
widely spread highly resistant haplotypes including quintuple (CIRNI-SGEAA) and sextuple (CIRNI-SGEGA) are likely to compromise effectiveness of IPTp-SP strategy. Continuous surveillance of SP effectiveness using molecular markers is therefore, critical and should be routinely implemented as recommended by WHO [29].

Determination of molecular markers in the Pfdhfr and Pfdhps genes offers an invaluable tool for epidemiological surveillance of SP resistance in malaria endemic settings and will generate important data to assist and inform malaria control programmes on the status of resistance particularly due to emergence and rapid spread of highly resistant mutations and haplotypes that may highly compromise the usefulness of IPT strategies. This study aimed to assess the status of Pfdhfr-Pfdhps mutations and haplotypes in areas with different malaria transmission intensities in mainland Tanzania, 6 years after withdrawal of SP as first line drug for treatment of uncomplicated falciparum malaria.

\section{Methods}

\section{Study sites}

The samples were collected from cross-sectional surveys at three sites with heterogeneous malaria transmission intensities in Tanzania from May to August 2013. The sites included Mkuzi health Centre in Muheza District in Tanga Region, Rubya Designated District Hospital (DDH), Muleba District in Kagera Region and Nachingwea District hospital in Lindi Region. The Regions are located in north-eastern, north-western and southern zones of mainland Tanzania, respectively (Fig. 1). The study districts were selected based on evidence from the Tanzania HIV and Malaria Indicator Survey of 2012 and represent areas with low, moderate and high malaria transmission intensities [30].

\section{Study design and sampling methods}

Cross-sectional studies were carried out in the period May-August 2013. Briefly, patients aged $\geq 6$ months who

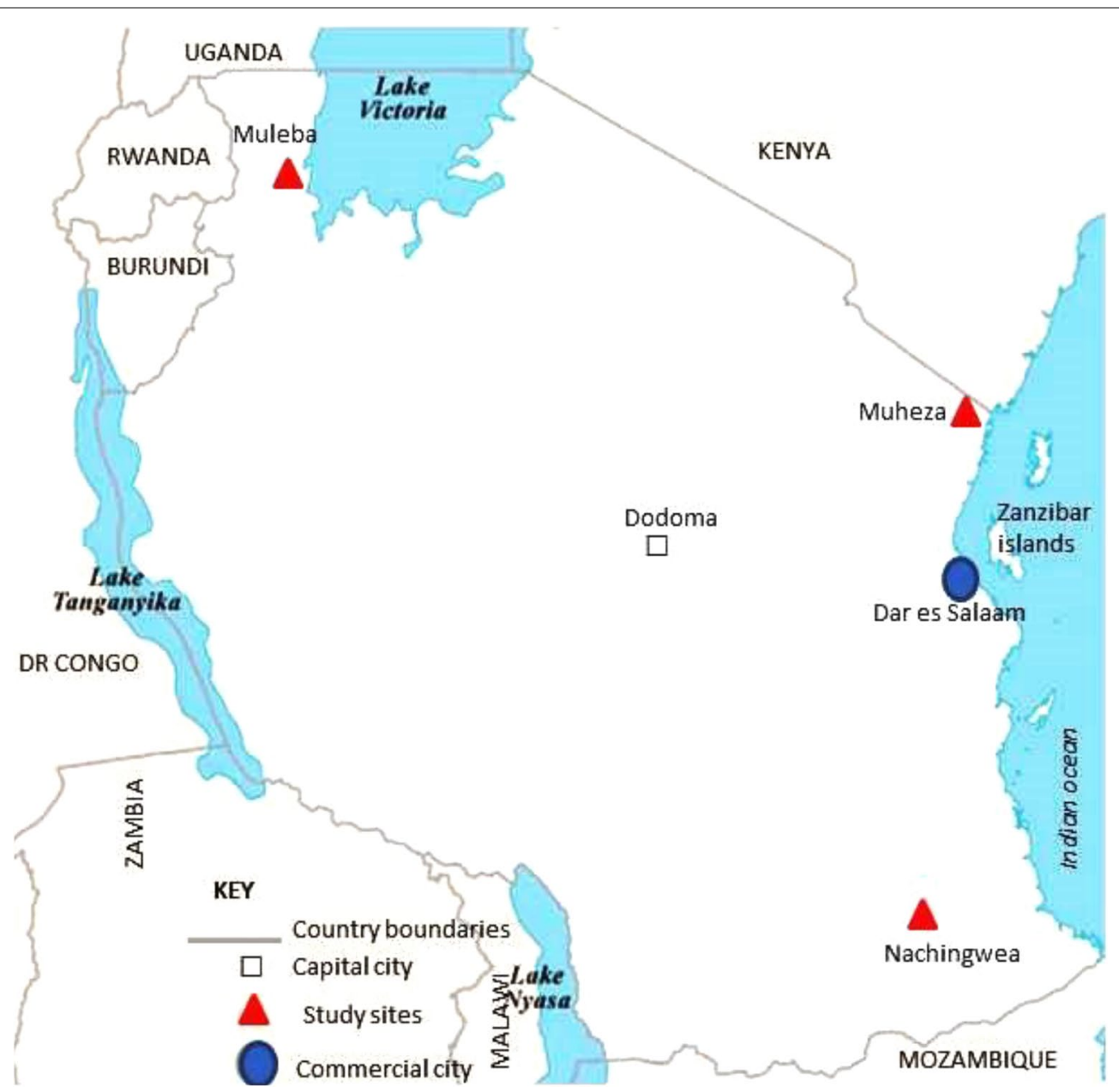

Fig. 1 The map of Tanzania showing the study site and neighbouring countries 
presented at the health facilities with uncomplicated or complicated malaria were enrolled. Patients with history of fever in the past $24 \mathrm{~h}$ or fever at presentation (temperature $\geq 37.5^{\circ} \mathrm{C}$ ) were screened for malaria and their demographic, clinical and parasitological information were collected. Laboratory screening for malaria was done using rapid diagnostic tests (RDT) and confirmed using microscopic examination of blood smears. Venous blood samples (3-5 ml) were collected into EDTA tubes for genomic analysis of malaria parasites. Thick and thin blood smears were prepared for detection and quantification of malaria parasites. The smears were air-dried and the thin films were fixed with methanol prior to staining using $3 \%$ Giemsa stain for $45 \mathrm{~min}$. Slides were examined microscopically to count asexual or sexual parasites per 200 white blood cells (WBCs) or 500WBCs, respectively. A slide was considered negative if no parasite was detected after examining 200 fields. Parasite density was calculated by multiplying the number of asexual parasites by 40 and sexual parasites by 16 assuming that one microliter of blood contained 8000 WBCs. Haemoglobin measurement was done using a Hemocue ${ }^{\circledR}$ machine (HemoCue, Ångelholm, Sweden). All patients who tested microscopically positive for malaria were treated as per national guidelines for treatment of malaria [31]. Global positioning system (GPS) coordinates were recorded using a handheld GPS device. In Muheza District, Tanga Region, samples were also collected from a clinical trial evaluating the efficacy of AL as per study protocol [32].

\section{DNA extraction}

Blood smear microscopically positive $P$. falciparum infected blood samples were used for DNA extraction. The samples were depleted of the white blood cells (WBCs) using a CF11 cellulose columns as previously described [33]. Genomic DNA was extracted using the QIAamp DNA Blood Midi (Qiagen GmbH, Hilden, Germany) as per manufacturer's instructions.

\section{Genotyping for Pfdhps and Pfdhfr mutations}

The SNPs in the Pfdhfr and Pfdhps genes were detected by a combination of nested PCR and sequence specific oligonucleotide probe (SSOP)-ELISA analysis as previously described [34]. The SNPs analysed were Pfdhfr at codons C50R, N51I, C59R, S108N, and L164I, and Pfdhps at codons S436A, A437G, K540E, A581G and A613S.

\section{Ethical issues}

The studies which contributed specimens were approved by the Tanzanian Medical Research Coordinating Committee of (MRCC) hosted by the National Institute for Medical Research, Tanzania (NIMR). Permission to conduct the study in the three districts was obtained from the relevant regional and district authorities while the heads of the health facilities gave the permission to conduct the study at the respective sites. Prior to enrolment, written informed consent was obtained from each individual or from parents/legally acceptable representative in case of children.

\section{Data analysis}

Microsoft Access version 2007 and Excel databases were utilized for data management with double entry, validation and cleaning of the field and laboratory data. Statistical analyses were done using STATA version 11 (STATA Corp Inc., TX, USA). Characteristics of the study population for the different sites were tested by analysis of variance (ANOVA). The prevalence of the genotypes was calculated as the proportion of wild type, mixed or mutants in the total analysable samples. Mixed infections with wild-type and mutant alleles were treated as mutant while generating the haplotype prevalence. Prevalence of mutations and haplotypes between sites were compared using Chi-square or Fisher's exact test as appropriate. Statistical analyses were performed at the $5 \%$ significance level and $95 \%$ Confidence Interval (CI).

\section{Results}

A total of 264 samples from the three sites (Muheza, Muleba and Nachingwea) were included in the analysis with each site contributing 88 samples. The demographic and parasitological characteristics of the study population between the study sites were comparable (Table 1). The study populations were similar with respect to age $\left(x^{2}=3.71, p=0.16\right)$, gender $\left(x^{2}=1.92, p=0.38\right)$ and the mean haemoglobin levels $(\mathrm{g} / \mathrm{dL})(\mathrm{F}=2.5, \mathrm{p}=0.08)$. However, the geometric mean parasite density was significantly lower at Muleba compared to Muheza and Nachingwea $(\mathrm{F}=10.9, \mathrm{p}<0.001)$, (Table 1$)$. Fever at health facility presentation (temperature $\geq 37.5{ }^{\circ} \mathrm{C}$ ) was significantly higher at Muheza $\left(\mathrm{x}^{2}=8.37, \mathrm{p}=0.02\right)$ compared to Muleba and Nachingwea. Only a few patients reported prior history of AL $(n=13)$ or SP $(n=2)$ usage 2 weeks prior to enrolment into the study.

\section{Prevalence of SNPs associated with SP resistance in Plasmodium falciparum dhfr and dhps genes}

Significantly higher prevalence of Pfdhfr mutations at codons C50/N51I was detected at Muheza (100\%) and Muleba (98.8 \%) compared to Nachingwea (67.5 \%), ( $\mathrm{p}<0.001)$. At Muheza, the Pfdhfr mutation at codon C59즈 had reached almost saturation level $(96.6 \%)$ while other sites, Muleba (73.1\%) and Nachingwea (81.8\%), had significantly lower prevalence $(\mathrm{p}<0.001)$. For codon S108N, the prevalence was significantly higher at Muheza $(98.7 \%)$ and Nachingwea (95.5 \%) compared to Muleba 
Table 1 Demographic and parasitological characteristics of the study population in Tanzania

\begin{tabular}{|c|c|c|c|c|}
\hline \multirow[t]{2}{*}{ Parameters } & \multicolumn{3}{|l|}{ Study site } & \multirow[t]{2}{*}{$P$ value } \\
\hline & Muheza $(n=88)$ & Muleba $(n=88)$ & Nachingwea $(\mathrm{n}=88)$ & \\
\hline Median age (years) (25-75 \% IQR) & $4.2(2.1-6.3)$ & $4.4(2.2-13.0)$ & $5.2(2.9-12.6)$ & 0.16 \\
\hline Sex, female n (\%) & $42(47.7)$ & $48(54.5)$ & $51(58.0)$ & 0.38 \\
\hline Mean haemoglobin $(\mathrm{g} / \mathrm{dL}),(\mathrm{SD})^{\mathrm{a}}$ & $10.4(1.8)$ & $9.9(2.4)$ & $9.7(2.4)$ & 0.08 \\
\hline GMPD $(95 \% C l)^{a}$ & $18,603(13,280-26,060)$ & $3700(1899-7211)$ & $12,968(8066-20,848)$ & $<0.001$ \\
\hline Fever at presentation $\left(\geq 37.5^{\circ} \mathrm{C}\right), \mathrm{n}(\%)$ & $65(73.9)$ & $59(67.1)$ & $47(53.4)$ & 0.02 \\
\hline Antimalarial treatment history, n (\%) & NA & $10(11.4)$ & $5(6.2)$ & 0.23 \\
\hline
\end{tabular}

Significant values are in boldface

$I Q R$ inter-quartile range, GMPD geometric mean parasite density, asexual parasites $/ \mu \mathrm{L}$ of blood

a One way ANOVA was used to test the differences between the study sites

$(83.3 \%)(\mathrm{p}=0.003)$. The Pfdhfr mutation at codon I164 $\underline{\mathbf{L}}$ was not detected at any site.

For the Pfdhps, significantly high prevalence of parasite carrying mutant at codons S436A/A437 G was detected $(79.8 \%)$ at Muheza as compared to Muleba (22.2\%) while Nachingwea had none $(\mathrm{p}<0.001)$. However, Nachingwea had significantly higher prevalence $(61.4 \%)$ of double mutants S436ㅁ/A437 G ( $\mathrm{p}<0.001)$. The prevalence of Pfdhps K540E at Muheza was significantly higher (95.4\%) compared to the other sites $(\mathrm{p}<0.001)$. Another Pfdhps mutation also associated with high level resistance at codon A581 $\underline{\mathbf{G}}$ was significantly higher at Muheza ( $\mathrm{p}<0.001$ ) reaching $48.9 \%$ in comparison to Muleba (3.9\%) and Nachingwea at which the mutations was not detected. Low prevalence of Pfdhps A613ㅍ was detected at Muleba (2/77, $2.6 \%)$ and Nachingwea (1/82, $1.2 \%)$.

\section{Prevalence of the major Plasmodium falciparum dhfr and dhps haplotypes}

Similarly, the prevalence of double mutant haplotypes (CICNI, CNㅌNI and CIRSI) was low except for Muleba where a significantly higher prevalence $(23.6 \%)$ of double haplotype CICNI was reported compared to the other sites $(\mathrm{p}=0.008)$. Overall, the triple Pfdhfr mutants (CIRNI) were predominant at all sites and almost near saturation at Muheza (93.3\%) but with significantly lower prevalence of and at Muleba (75\%) and Nachingwea $(70.6 \%)$, $(\mathrm{p}<0.001)$.

The prevalence of wildtype Pfdhps haplotype (SAKAA) was significantly low at Muheza (1.3\%) compared to other sites $(\mathrm{p}=0.003)$. Similarly, the three major single mutant haplotypes were also low (Table 2). The double Pfdhps haplotype SGEAA was higher at Muheza (27.2\%) and Muleba $(20.8 \%)$ compared to Nachingwea $(\mathrm{p}<0.001)$ while $\underline{\mathbf{A} A K A} \underline{\mathbf{S}}$ was low at both site $(\mathrm{p}=0.76)$. About $56 \%$ of all isolates were triple mutants with Muheza having the highest prevalence of SGEGA haplotype compared to AGEAA which was more predominant at Muleba and Nachingwea ( $<0.001)$, (Table 2). The Pfdhps quadruple haplotypes AGEGA were generally low and varied significantly between the sites, whereby eight isolates (10.4\%) were detected at Muheza, two at Muleba (5.6\%) and none was detected at Nachingwea $(\mathrm{p}=0.003)$ (Table 2$)$.

Upon combination of the Pfdhfr-Pfdhps haplotypes (Table 3), quadruple mutant haplotypes with single Pfdhps and triple Pfdhfr mutation ( lowly distributed $(\mathrm{n}=16)$ across all sites while the quintuple mutant haplotype, CIRNI-SGEAA was observed in only 31 isolates, at Muheza and Muleba. Sextuple CIRNISGEGA haplotypes $(\mathrm{n}=32)$ were more predominant in Muheza whereas other sextuple haplotype combinations, (CIRNI-AGEAA $(\mathrm{n}=69)$ ) were mainly observed in Nachingwea. Interestingly, the emergence of the new septuple mutant haplotypes with three Pfdhfr and four Pfdhps mutant combination (CIRNI-AGEGA) was observed for the first time albeit in few samples $(n=11)$ and these were mainly from Muheza $(n=8)$ and $(n=3)$ were from Muleba whereas none was noted at Nachingwea. The occurrences of other Pfdhps-Pfdhfr haplotypes were generally low (Table 3 ).

\section{Discussion}

The rapid and widespread anti-malarial drug resistance has necessitated frequent revisions of malaria treatment guidelines in $P$. falciparum malaria endemic regions. The emergence of super resistant mutations, such as the sextuple Pfdhfr/Pfdhps haplotypes has not only compromised malaria case management and treatment outcomes but also affected the effectiveness of the IPT strategies.

Few years after its adoption as the first-line treatment drug for uncomplicated malaria in Tanzania, several efficacy studies detected unacceptably high levels of molecular markers of parasite resistance to SP $[4,35]$. Despite its replacement by $\mathrm{ACT}$, SP continued to be used in the IPT 
Table 2 P. falciparum dihydrofolate reductase (Pfdhfr) and dihydropteroate synthetase (Pfdhps) haplotypes by study sites

\begin{tabular}{|c|c|c|c|c|c|}
\hline \multicolumn{2}{|c|}{ Gene haplotype } & \multicolumn{4}{|c|}{ Prevalence of haplotypes by sites } \\
\hline & & Muheza $(n=77)$ & Muleba $(n=72)$ & Nachingwea $(n=85)$ & $P$ value \\
\hline \multirow[t]{6}{*}{ Pfdhfr } & CNCSI & $0(0)$ & $0(0)$ & $3(3.5)$ & 0.109 \\
\hline & $C|\underline{C}|$ & $4(5.2)$ & $17(23.6)$ & $7(8.4)$ & 0.002 \\
\hline & $\mathrm{C} \underline{\mathbf{I R}} \mathrm{SI}$ & $1(1.3)$ & $0(0)$ & $0(0)$ & 0.642 \\
\hline & CNR & $0(0)$ & $1(1.4)$ & $15(17.7)$ & $<0.001$ \\
\hline & CIRN & $72(93.3)$ & $54(75.0)$ & $60(70.6)$ & $<0.001$ \\
\hline & & $n=77$ & $n=72$ & $\mathrm{n}=82$ & \\
\hline \multirow[t]{9}{*}{ Pfdhps } & SAKAA & $1(1.3)$ & $11(15.3)$ & $11(16.5)$ & 0.003 \\
\hline & $\underline{\mathbf{A}} \mathrm{AKAA}$ & $4(5.2)$ & $4(5.6)$ & $16(18.8)$ & 0.008 \\
\hline & SAKA $\underline{\mathbf{S}}$ & $0(0)$ & $1(1.4)$ & $0(0)$ & 0.308 \\
\hline & SAEAA & $0(0)$ & $4(5.6)$ & $0(0)$ & 0.008 \\
\hline & $\underline{\mathbf{A}} \mathrm{AKA} \underline{\mathbf{S}}$ & $0(0)$ & $1(1.4)$ & $1(1.2)$ & 0.76 \\
\hline & SGEAA & $21(27.2)$ & $15(20.8)$ & $0(0)$ & $<0.001$ \\
\hline & SGEGA & $31(40.3)$ & $2(2.8)$ & $0(0)$ & $<0.001$ \\
\hline & AGEAA & $12(15.6)$ & $30(41.7)$ & $54(63.5)$ & $<0.001^{*}$ \\
\hline & AGEGA & $8(10.4)$ & $4(5.6)$ & $0(0)$ & 0.003 \\
\hline
\end{tabular}

Bold underline indicates the amino acid changes

$n$ number of haplotypes excluding the mixed infections/genotypes

* Pearson Chi-square test was applied $(x 2=39.9, \mathrm{p}<0.001)$, all others were compared using Fisher's exact test

Table 3 The pattern of combined P. falciparum dhfr-dhps haplotypes

\begin{tabular}{|c|c|c|c|c|c|c|c|}
\hline \multicolumn{2}{|c|}{ Pfdhps haplotypes } & \multicolumn{5}{|c|}{ Pfdhfr haplotypes } & \multirow[t]{3}{*}{ Total } \\
\hline & & \multirow{2}{*}{$\begin{array}{l}\text { Wild-type } \\
\text { CNCSI }\end{array}$} & \multicolumn{3}{|c|}{ Double } & \multirow{2}{*}{$\begin{array}{l}\text { Triple } \\
\text { CIRNI }\end{array}$} & \\
\hline & & & CICNI & CIRSI & CNRNI & & \\
\hline Wildtype & SAKAA & 0 & 4 & 0 & 2 & 20 & 26 \\
\hline \multirow[t]{3}{*}{ Single } & $\underline{\mathbf{A}} \mathrm{AKAA}$ & 1 & 3 & 0 & 4 & 16 & 24 \\
\hline & SAEEAA & 0 & 0 & 0 & 0 & 4 & 4 \\
\hline & SAKA $\underline{\mathbf{S}}$ & 0 & 0 & 0 & 0 & 1 & 1 \\
\hline \multirow[t]{2}{*}{ Double } & S $\underline{\text { GEAA }}$ & 0 & 5 & 0 & 0 & 31 & 36 \\
\hline & $\underline{\mathbf{A}} \mathrm{AKA} \underline{\mathbf{S}}$ & 0 & 0 & 0 & 0 & 2 & 2 \\
\hline \multirow[t]{2}{*}{ Triple } & S $\underline{\mathbf{G E G}} \mathrm{A}$ & 0 & 1 & 0 & 0 & 32 & 33 \\
\hline & AGEAA & 2 & 14 & 1 & 10 & 69 & 96 \\
\hline Quadruple & AGEGA & 0 & 1 & 0 & 0 & 11 & 12 \\
\hline Total & & 3 & 28 & 1 & 16 & 186 & 234 \\
\hline
\end{tabular}

The overall numbers (absolute) parasite haplotypes on the Pfdhfr-Pfdhps genes in the samples successfully analysed ( $n=234)$. Of all the combined haplotypes, the quintuple CIRNI-SGEAA, sextuple CIRNI-AGEAA, CIRNI-SGEGA were more frequently detected

Bold underline indicates the amino acid changes, while SAKAA and CNCSI represent the wildtype haplotype for Pfdhps and Pfdhfr, respectively

strategies which is likely to provide sub-optimal effect and, therefore, monitoring the spread of resistance using molecular markers (Pfdhfr/Pfdhps) is still recommended [36-38]. In this study, the prevalence of A581 $\underline{\mathbf{G}}$ was almost $50 \%$ in Muheza and this was significantly higher compared to Muleba ( 4\%) and Nachingwea (0\%). This could confirm the earlier suggestions that this "super resistant" mutation may have originated in the northeastern part of Tanzania and spread to other areas albeit at low prevalence [37].

A remarkable difference was observed in the prevalence of Pfdhps SNPs between the study sites with Muheza having the highest levels of Pfdhps 436/437 $\underline{\text { SG, }}$ A581ㅁ, A540E SNPs and haplotypes compared to 
Muleba and Nachingwea. As expected areas with high malaria transmission intensities in Muleba and Nachingwea had higher prevalence of Pfdhps wild types and sin-

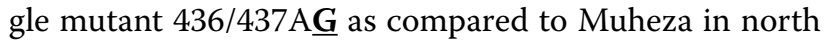
eastern where malaria endemicity has declined remarkably in recent years $[39,40]$. The current WHO recommendations suggest that SP-IPTp should be discontinued if the prevalence of this double Pfdhps mutant, K540E is more than $95 \%$ and the A581 $\mathbf{G}$ is more than $10 \%$ as it is considered to be ineffective [29]. Obviously, these criteria are still met in Muheza confirming the findings of previous studies conducted in north Eastern Tanzania [26]. In a cohort study conducted at Muheza, it was shown that IPTp-SP was associated with increased prevalence of parasites with mutations at codon A581 $\underline{\mathbf{G}}$ and that use of IPTp-SP conferred no benefit in improvement of pregnancy outcomes $[17,37]$. Of note, this area is known to be the major focus of Pfdhps A581G mutations in East Africa which is believed to occur almost exclusively with Pfdhfr K540E leading to double mutant haplotypes [41]. The high frequency of Pfdhps A581G at alarming level in this area clearly suggests for no beneficial protective effect from the IPTp-SP $[17,18,42]$. This higher prevalence in north eastern Tanzania could be explained by the sustained drug pressure due to self-medication that could have elevated levels of SP resistance, Ringsted et al. [43] reported $76 \%$ volume sales of SP in private drug shops in this areas. Additionally, the high prevalence levels could be maintained due to selective pressure on $P f d h f r$ and Pfdhps as a result of co-trimoxazole (trimethoprimsulfamethoxazole), another antifolate, used to prevent opportunistic infections in HIV infected individuals as cross-resistance might also occur $[44,45]$.

The prevalence of the Pfdhfr-Pfdhps wild type haplotypes was low in all sites. The prevalence of mutant genotypes C51I, was at saturation in Muheza (100\%) and equally at Muleba (98.8\%) with significantly lower prevalence $(67.5 \%)$ at Nachingwea $(\mathrm{p}<0.001)$. Similarly, almost complete saturation ( $>96 \%$ ) of other muta-

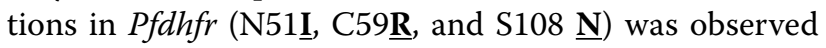
at Muheza with marked differences between Muleba and Nachingwea (Table 2). In contrast, some previous studies have shown that other major resistance mutations in Pfdhfr are well established throughout the country where

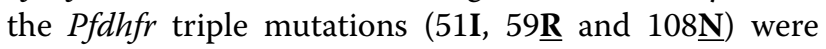
above $84 \%$ and close to saturation in six regions of Tanzania [46]. Elsewhere, these data are in consistent with studies in West Africa where Pfdhfr N51, N59 $\underline{\mathbf{R}}$, and S108N have been shown to rise [19]. The prevalence of I164L mutation conferring high pyrimethamine resistance was not detected at all the three sites which is in accordance to previous reports elsewhere [47]. However, in other parts of Africa, only a few studies have reported occurrence of this high level mutation $[15,16,48]$. Of note, the Pfdhfr I164L mutation was first documented at low prevalence in Muheza in 1999 before the deployment of IPTp-SP [49] and later one isolate (mixed allele) was reported in Rufiji [50], but to date its presence has rarely been reported in the country.

The CIRNI haplotype with triple mutations was present and highly prevalent at all study sites regardless of the transmission intensity. This is in line with several studies in Tanzania and elsewhere in the SSA [51, 52]. The CIRNI haplotype is associated with high level resistance to pyrimethamine and increases the risk of SP resistance if it occurs concurrently with Pfdhps mutations [21]. The increased double mutant Pfdhps SGEAA haplotype was observed in Muheza (28\%) and Muleba (22\%), but not in Nachingwea (0\%). In Muheza, the highly resistant triple SGEGA Pfdhps haplotypes was observed, (38.7 \%).

The combinations of Pfdhfr-Pfdhps were detected at higher numbers including quintuple mutant CIRNISGEAA, sextuple haplotype which comprise triple mutations in both genes CIRNI-SGEGA and CIRNI-AGEGA have been highly associated with sub-optimal IPTp-SP effectiveness in previous studies [17]. Interestingly, a septuple mutant haplotype CIRNI-AGEGA was observed which had not been previously reported in the study areas. From these observations, it is apparent that these mutant haplotypes associated with poor IPTp-SP are expanding in different epidemiological settings. Of worth noting, this study was not designed to correlate the clinical data with observed resistance pattern. Nonetheless previous literature showing the association of these haplotypes with clinical or treatment outcomes have been noted.

Ideally, in order to reduce the sustained drug pressure, efforts are required to limit the use of SP for IPTp purposes only through limiting over the counter SP prescriptions. Also the availability of ACT for the treatment of uncomplicated malaria and proper implementation of the national malaria treatment guidelines would also contribute in the reducing the selection pressure. However, alternative drugs for IPTp are urgently needed to replace the failing SP due to the saturation of the parasite population with Pfdhps-Pfdhfr mutations and haplotypes highly associated with SP resistance. Deployment of the suboptimal IPTp-SP strategy is therefore unlikely to confer the anticipated effect on improving pregnancy outcomes.

\section{Conclusion}

These results ascertain the high prevalence of $P f d h f r$ and Pfdhps haplotypes conferring SP resistance in areas with changing malaria epidemiology. The high prevalence of Pfdhfr-Pfdhps sextuple mutant haplotypes and the occurrence of the new septuple mutant haplotype undermine 
the usefulness of IPTp-SP. Thus, additional control efforts are needed to contain the spread of SP resistance and suitable alternative drugs for IPTp are urgently needed.

\section{Authors' contributions}

VB, RAM, DSI and JPG designed the study; VB, RAM, DN, DSI and JPG performed the research; VB, RAM, DTRM and FF analysed data; all authors contributed to the writing of the paper. All authors read and approved the final manuscript.

\section{Author details}

${ }^{1}$ National Institute for Medical Research, Tanga Research Centre, P. O. Box 5004, Tanga, United Republic of Tanzania. ${ }^{2}$ International Health Unit, Department of Epidemiology, University of Antwerp, Campus Drie Eiken, Universiteitsplein 1, 2610 Wilrijk, Belgium. ${ }^{3}$ Tanzania Food and Drugs Authority, P: O. Box 77150, Dar es Salaam, United Republic of Tanzania.

\section{Acknowledgements}

We thank Lydia Lugomora (NIMR), Gwen Lemey and Jan Vervoort (Universiteit Antwerpen, Belgium), Michael Alifrangis, Ulla Abildtrup and Christentze Schmiegelow (CMP-Denmark) for their logistical assistance. Our thanks also extend to the staff at AMBRELA laboratory who participated in field data collection and data management. The study was supported by the South Initiative Grant (Code ZAIN2014Z168) and ICP PhD Grant by the VLIR UOS, Brussels, Belgium (for the laboratory component) while the sample and data collection was supported by the UK Medical research Council (MRC) (G0600718-Centennial award).

\section{Competing interests}

The authors declare that they have no competing interests.

\section{Received: 26 February 2015 Accepted: 29 October 2015}

Published online: 05 November 2015

\section{References}

1. Griffin JT, Ferguson NM, Ghani AC. Estimates of the changing age-burden of Plasmodium falciparum malaria disease in sub-Saharan Africa. Nat Commun. 2014:5:3136.

2. Dondorp AM, Yeung S, White L, Nguon C, Day NPJ, et al. Artemisinin resistance: current status and scenarios for containment. Nat Rev Microbiol. 2010;8:272-80.

3. Ariey F, Witkowski B, Amaratunga C, Beghain J, Langlois A-C, Khim N, et al. A molecular marker of artemisinin-resistant Plasmodium falciparum malaria. Nature. 2014;505:50-5.

4. Mugittu K, Abdulla S, Falk N, Masanja H, Felger I, Mshinda H, et al. Efficacy of sulfadoxine-pyrimethamine in Tanzania after two years as first-line drug for uncomplicated malaria: assessment protocol and implication for treatment policy strategies. Malar J. 2005:4:55.

5. Wongsrichanalai C, Pickard AL, Wernsdorfer WH, Meshnick SR. Epidemiology of drug-resistant malaria. Lancet Infect Dis. 2002;2:209-18.

6. Wellems TE, Plowe CV. Chloroquine-Resistant Malaria. J Infect Dis. 2001;184:770-6.

7. WHO. Guideline for the treatment of malaria. 2nd edn. Geneva: World Health Organization; 2010

8. Policy M, Committee A. Malaria Policy Advisory Committee to the WHO conclusions and recommendations of March 2013 meeting. Malar J. 2013;12:213.

9. WHO. Seasonal Malaria Chemoprevention (SMC) for Plasmodium falciparum control in highly seasonal transmission areas of Sahel sub-region in Africa. Geneva. World Health Organization, 2012.

10. Mulligan JA, Mandike R, Palmer N, Williams H, Abdulla S, Bloland P, et al. The costs of changing national policy: lessons from malaria treatment policy guidelines in Tanzania. Trop Med Int Health. 2006;11:452-61.

11. Mugittu K. Therapeutic efficacy of sulfadoxine-pyrimethamine and prevalence of resistance markers in Tanzania prior to revision of malaria treatment policy. Trop Med Int Health. 2007;12:219-23.

12. Mugittu K, Genton B, Mshinda H, Beck HP. Molecular monitoring of Plasmodium falciparum resistance to artemisinin in Tanzania. Malar J. 2006;5:126.
13. Brooks DR, Wang P, Read M, Watkins WM, Sims PF, Hyde JE. Sequence variation of the hydroxymethyldihydropterin pyrophosphokinase: dihydropteroate synthase gene in lines of the human malaria parasite Plasmodium falciparum, with differing resistance to sulfadoxine. Eur J Biochem. 1994;224:397-405

14. Triglia T, Menting JG, Wilson C, Cowman AF. Mutations in dihydropteroate synthase are responsible for sulfone and sulfonamide resistance in Plasmodium falciparum. Proc Natl Acad Sci U S A. 1997;94:13944-9.

15. Lynch C, Pearce R, Pota H, Cox J, Abeku TA, Rwakimari J, et al. Emergence of a dhfr mutation conferring high-level drug resistance in Plasmodium falciparum populations from southwest Uganda. J Infect Dis. 2008;197:1598-604.

16. McCollum AM, Poe AC, Hamel M, Huber C, Zhou Z, Shi YP, et al. Antifolate resistance in Plasmodium falciparum: multiple origins and identification of novel dhfr alleles. J Infect Dis. 2006;194:189-97.

17. Minja DTR, Schmiegelow C, Mmbando B, Boström S, Oesterholt M, Magistrado $P$, et al. Plasmodium falciparum mutant haplotype infection during pregnancy associated with reduced birthweight. Tanzania. Emerg Infect Dis. 2013;19:1446-54.

18. Harrington WE, Morrison R, Fried M, Duffy PE. Intermittent preventive treatment in pregnant women is associated with increased risk of severe malaria in their offspring. PLoS ONE. 2013;8:e56183.

19. Naidoo I, Roper C. Mapping "partially resistant", "fully resistant", and "super resistant" malaria. Trends Parasitol. 2013;29:505-15.

20. McCollum AM, Mueller K, Villegas L, Udhayakumar V, Escalante AA. Common origin and fixation of Plasmodium falciparum dhfr and dhps mutations associated with sulfadoxine-pyrimethamine resistance in a low-transmission area in South America. Antimicrob Agents Chemother. 2007:51:2085-91.

21. Kublin JG, Dzinjalamala FK, Kamwendo DD, Malkin EM, Cortese JF, Martino LM, et al. Molecular markers for failure of sulfadoxine-pyrimethamine and chlorproguanil-dapsone treatment of Plasmodium falciparum malaria. J Infect Dis. 2002:185:380-8.

22. Triglia T, Wang P, Sims PF, Hyde JE, Cowman AF. Allelic exchange at the endogenous genomic locus in Plasmodium falciparum proves the role of dihydropteroate synthase in sulfadoxine-resistant malaria. EMBO J. 1998; 17:3807-15.

23. Vinayak S, Alam MT, Mixson-Hayden T, McCollum AM, Sem R, Shah NK, Lim P, Muth S, Rogers WO, Fandeur T, Barnwell JW, Escalante AA, Wongsrichanalai C, Ariey F, Meshnick SR, Udhayakumar V. Origin and evolution of sulfadoxine resistant Plasmodium falciparum. PLoS Pathog. 2010;6:e1000830.

24. Matondo SI, Temba GS, Kavishe AA, Kauki JS, Kalinga A, Van Zwetselaar $M$, Reyburn H, Kavishe RA. High levels of sulphadoxine-pyrimethamine resistance Pfdhfr-Pfdhps quintuple mutations: a cross sectional survey of six regions in Tanzania. Malar J. 2014;13:1-7.

25. Juma DW, Omondi AA, Ingasia L, Opot B, Cheruiyot A, Yeda R, et al. Trends in drug resistance codons in Plasmodium falciparum dihydrofolate reductase and dihydropteroate synthase genes in Kenyan parasites from 2008 to 2012. Malar J. 2014;13:250.

26. Gesase S, Gosling RD, Hashim R, Ord R, Naidoo I, Madebe R, et al. High resistance of Plasmodium falciparum to sulphadoxine/pyrimethamine in northern Tanzania and the emergence of dhps resistance mutation at codon 581. PLOS ONE. 2009;4:e4569.

27. Harrington WE, Mutabingwa TK, Kabyemela E, Fried M, Duffy PE. Intermittent treatment to prevent pregnancy malaria does not confer benefit in an area of widespread drug resistance. Clin Infect Dis. 2011:53:224-30.

28. WHO. WHO Evidence Review Group on Intermittent Preventive Treatment (IPT) of malaria in pregnancy, Geneva. World Health Organization, 2013.

29. WHO. WHO Policy Brief for the Implementation of Intermittent Preventive Treatment of Malaria in Pregnancy., Geneva. World Health Organization, 2013

30. National Bureau of Statistics. 2011-2012 Tanzania HIV/AIDS and Malaria Indicator Survey. Dar es Salaam: NBS; 2012.

31. MoHSW. National Guidelines for malaria diagnosis and treatment. Ministry of Health: United Republic of Tanzania; 2006.

32. Shayo A, Mandara Cl, Shahada F, Buza J, Lemnge MM, Ishengoma DS. Therapeutic efficacy and safety of artemether-lumefantrine for the treatment of uncomplicated falciparum malaria in North-Eastern Tanzania. Malar J. 2014:13:376. 
33. Venkatesan M, Amaratunga C, Campino S, Auburn S, Koch O, Lim P, et al. Using CF11 cellulose columns to inexpensively and effectively remove human DNA from Plasmodium falciparum-infected whole blood samples. Malar J. 2012;11:41.

34. Alifrangis M, Enosse S, Pearce R, Drakeley C, Roper C, Khalil IF, et al. A simple, high-throughput method to detect Plasmodium falciparum single nucleotide polymorphisms in the dihydrofolate reductase, dihydropteroate synthase, and P. falciparum chloroquine resistance transporter genes using polymerase chain reaction- and enzy. Am J Trop Med Hyg. 2005;72:155-62

35. Pearce RJ, Drakeley C, Chandramohan D, Mosha F, Roper C. Molecular determination of point mutation haplotypes in the dihydrofolate reductase and dihydropteroate synthase of Plasmodium falciparum in three districts of Northern Tanzania. Antimicrob Agents Chemother. 2003;47:1347-54.

36. Alifrangis M, Lusingu JP, Mmbando B, Dalgaard MB, Vestergaard LS, Ishengoma D, et al. Five-Year Surveillance of Molecular Markers of Plasmodium falciparum Antimalarial Drug Resistance in Korogwe District, Tanzania: accumulation of the $581 \mathrm{G}$ Mutation in the P. falciparum Dihydropteroate Synthase Gene. Am J Trop Med Hyg. 2009;80:523-7.

37. Alifrangis M, Nag S, Schousboe ML, Ishengoma D, Lusingu J, Pota H, et al. Independent origin of plasmodium falciparum antifolate super-resistance, Uganda, Tanzania, and Ethiopia. Emerg Infect Dis. 2014;20:1280-6.

38. Malisa AL, Pearce RJ, Abdulla S, Mshinda H, Kachur PS, Bloland P, et al. Drug coverage in treatment of malaria and the consequences for resistance evolution-evidence from the use of sulphadoxine/pyrimethamine. Malar J. 2010;9:190.

39. Ishengoma DS, Mmbando BP, Segeja MD, Alifrangis M, Lemnge MM, Bygbjerg IC. Declining burden of malaria over two decades in a rural community of Muheza district, north-eastern Tanzania. Malar J. 2013;12:338.

40. Mmbando BP, Vestergaard LS, Kitua AY, Lemnge MM, Theander TG, Lusingu JPA. A progressive declining in the burden of malaria in northeastern Tanzania. Malar J. 2010;9:216.

41. Naidoo I, Roper C. Following the path of most resistance: dhps K540E dispersal in African Plasmodium falciparum. Trends Parasitol. 2010;26:447-56

42. Harrington WE, Mutabingwa TK, Muehlenbachs A, Sorensen B, Bolla MC, Fried M, et al. Competitive facilitation of drug-resistant Plasmodium falciparum malaria parasites in pregnant women who receive preventive treatment. Proc Natl Acad Sci U S A. 2009;106:9027-32.

43. Ringsted FM, Massawe IS, Lemnge MM, Bygbjerg IC. Saleability of antimalarials in private drug shops in Muheza, Tanzania: a baseline study in an era of assumed artemisinin combination therapy (ACT). Malar J. 2011;10:238.

44. Iyer JK, Milhous WK, Cortese JF, Kublin JG, Plowe CV. Plasmodium falciparum cross-resistance between trimethoprim and pyrimethamine. Lancet. 2001;358:1066-7.

45. Khalil I, Rønn AM, Alifrangis M, Gabar HA, Satti GMH, Bygbjerg IC, et al. Dihydrofolate reductase and dihydropteroate synthase genotypes associated with in vitro resistance of Plasmodium falciparum to pyrimethamine, trimethoprim, sulfadoxine, and sulfamethoxazole. Am J Trop Med Hyg. 2003;68:586-9.

46. Matondo SI, Temba GS, Kavishe AA, Kauki JS, Kalinga A, van Zwetselaar $M$, et al. High levels of sulphadoxine-pyrimethamine resistance PfdhfrPfdhps quintuple mutations: a cross sectional survey of six regions in Tanzania. Malar J. 2014;13:152.

47. Raman J, Little F, Roper C, Kleinschmidt I, Cassam Y, Maharaj R, et al. Five years of large-scale dhfr and dhps mutation surveillance following the phased implementation of artesunate plus sulfadoxine-pyrimethamine in Maputo Province, Southern Mozambique. Am J Trop Med Hyg. 2010;82:788-94.

48. Alker AP, Mwapasa V, Purfield A, Rogerson SJ, Molyneux ME, Kamwendo DD, et al. Mutations associated with sulfadoxine-pyrimethamine and chlorproguanil resistance in Plasmodium falciparum isolates from Blantyre. Malawi. Antimicrob Agents Chemother. 2005;49:3919-21.

49. Hastings MD, Bates SJ, Blackstone EA, Monks SM, Mutabingwa TK, Sibley $\mathrm{CH}$. Highly pyrimethamine-resistant alleles of dihydrofolate reductase in isolates of Plasmodium falciparum from Tanzania. Trans R Soc Trop Med Hyg. 2002;96:674-6

50. Malisa AL, Pearce RJ, Mutayoba BM, Abdullah S, Mshinda H, Kachur PS, et al. The evolution of pyrimethamine resistant dhfr in Plasmodium falciparum of south-eastern Tanzania: comparing selection under SP alone vs SP + artesunate combination. Malar J. 2011;10:317.

51. Nsimba B, Jafari-Guemouri S, Malonga DA, Mouata AM, Kiori J, Louya $\mathrm{F}$, et al. Epidemiology of drug-resistant malaria in Republic of Congo: using molecular evidence for monitoring antimalarial drug resistance combined with assessment of antimalarial drug use. Trop Med Int Health. 2005;10:1030-7.

52. Mombo-Ngoma G, Oyakhirome S, Ord R, Gabor JJ, Greutélaers KC, Profanter $\mathrm{K}$, et al. High prevalence of dhfr triple mutant and correlation with high rates of sulphadoxine-pyrimethamine treatment failures in vivo in Gabonese children. Malar J. 2011:10:123.

\section{Submit your next manuscript to BioMed Central and take full advantage of:}

- Convenient online submission

- Thorough peer review

- No space constraints or color figure charges

- Immediate publication on acceptance

- Inclusion in PubMed, CAS, Scopus and Google Scholar

- Research which is freely available for redistribution

Submit your manuscript at

www.biomedcentral.com/submit
C Biomed Central 